\title{
Neuropsychologische Schätzdefizite bei rechtsfrontaler und rechtstemporaler Epilepsie
}

\author{
Michael Schwarz, Edith Benkowitsch, Katrin Schneider, Katrin Walther und Hajo Hamer \\ Neurologische Universitätsklinik Erlangen, Deutschland
}

\begin{abstract}
Zusammenfassung: Hintergrund: Nach dem bisherigen Forschungsstand sind neuropsychologische Beeinträchtigungen der Schätzfunktion vor allem bei rechtshemisphärischen Läsionen zu erwarten. Ziele: Es wurde die Fähigkeit des Schätzens bei Epilepsiepatient_innen und einer gesunden Kontrollgruppe untersucht. Je nach Läsionsort wurden sechs Gruppen (rechtsfrontal, linksfrontal, rechtstemporal-mesial, rechtstemporal-neokortikal, linkstemporal-mesial, linkstemporal-neokortikal) einbezogen. Methoden: Mit 176 Personen, 109 Patient_innen und 67 Kontrollpersonen, wurde der Test zum kognitiven Schätzen durchgeführt. Fragestellung: Es wurde erwartet, dass speziell Patient_innen mit rechtshemisphärischen Läsionen Defizite im Schätzen aufweisen. Ergebnisse: Patient_innen mit rechtsfrontalen und rechtstemporalen mesialen Läsionen wiesen signifikant schlechtere Schätzleistungen gegenüber Patient_innen mit linkshemisphärischen Läsionen und der Kontrollgruppe auf. Fazit: Die Ergebnisse sprechen für die vorherrschende Beteiligung der rechten Hemisphäre an der Fähigkeit des kognitiven Schätzens.
\end{abstract}

Schlüsselwörter: Kognitives Schätzen, Epilepsie, Temporallappen, Frontallappen, Hemisphärenunterschiede, Hippocampus

Deficits in cognitive estimation in patients with right-frontal and right-temporal lobe epilepsy

Abstract: Background: Previous studies revealed a right-hemispheric dominance for cognitive estimation processes. Objectives: This study explored estimation impairments in patients with focal temporal and frontal lobe epilepsies. Methods: 109 patients (right frontal, left frontal, right temporal-mesial, right temporal-neocortical, left temporal-mesial, left temporal-neocortical) and a control group with 67 subjects were included. All subjects underwent an extensive neuropsychological assessment including the Test zum kognitiven Schätzen (TKS; Brand et al., 2002). Hypothesis: It was expected that right-hemispheric lesions would be associated with impairments in cognitive estimation. Results: Patients with right-frontal and right-temporal mesial focus showed significant estimation deficits compared to the other groups. Conclusion: This study shows that the right hemisphere dominantly participates in cognitive estimation.

Keywords: cognitive estimation, epilepsy, temporal lobe, frontal lobe, laterality, hippocampus

\section{Einleitung}

Bei Epilepsiepatient_innen liefern neuropsychologische Defizite wichtige Anhaltspunkte hinsichtlich der Lokalisation des epileptischen Fokus. Kognitive Funktionsstörungen äußern sich bei therapierefraktären Epilepsien typischerweise in einer mentalen Verlangsamung, Gedächtnisbeeinträchtigungen und Störungen der Aufmerksamkeitsfunktionen (van Rijckevorsel, 2006). Häufig sind in besonderer Weise die Exekutivfunktionen betroffen (Benke, Lehner-Baumgartner, Bodner, Karner \& Delazer, 2007), wobei dies insbesondere Patient_ innen mit Frontallappenepilepsie betrifft (Hernandez et al., 2002).
Allgemein umfassen exekutive Funktionen eine Vielzahl von Regulations- und Kontrollmechanismen zur Gewährleistung eines zielorientierten Verhaltens. Dazu zählen das Steuern der Aufmerksamkeit auf relevante Bereiche, das Entwickeln und Anwenden von Plänen, das Befolgen sozialer Regeln, das Lösen komplexer Aufgabenstellungen, das parallele Durchführen mehrerer Aufgaben, die Überwachung des Arbeitsgedächtnisses sowie die Verortung von Informationen in Raum und Zeit (Bunge \& Souza, 2009). Zusammengenommen können exekutive Funktionsdefizite bei den Betroffenen durch Störungen des Arbeitsgedächtnisses und Monitorings, der kognitiven Flexibilität und Flüssigkeit und des planerischen und problemlösenden Denkens beschrieben werden (Müller, Klein et al., 2019). 
Zum Spektrum der exekutiven Funktionen ist auch das kognitive Schätzen zu zählen. In der Literatur wird das kognitive Schätzen als eine Fähigkeit verstanden, Strategien des schlussfolgernden Denkens auf Fragen anzuwenden, für die es prinzipiell keine eindeutige und präzise Antwort gibt (D'Aniello, Scarpina, Albani, Castelnuovo \& Mauro, 2015). Analog definieren Brand et al. (2002) das Schätzen als einen Prozess der Antwortgenerierung, wobei die Lösung mithilfe des semantischen Wissens oder der Anwendung einer Vergleichsstrategie nicht direkt verfügbar ist. Für viele Alltagssituationen ist das angemessene Schätzen von physischen und metrischen Proportionen sehr wichtig. Entsprechend ist auch das Wissen um die Lokalisation dieser Funktion im menschlichen Gehirn neurowissenschaftlich bedeutsam (Braun et al., 2018).

Zahlreiche Studien zeigen für das Schätzen eine dominante funktionelle Zuständigkeit des Frontallappens (Helmstaedter, Kemper \& Elger, 1996; Shallice, 1982; Shallice \& Evans, 1978; Upton \& Thompson, 1996; Lewis \& Miall, 2006; Morin et al., 2005). Smith und Milner (1984) untersuchten Patient innen mit unilateralen frontalen oder temporalen operativen Eingriffen im Gehirn und verglichen diese mit einer gesunden Kontrollgruppe. Sie fanden heraus, dass Patient_innen nach rechtsfrontalen Lobektomien im Vergleich zu Patient_innen mit temporaler Resektion deutliche Beeinträchtigungen beim Schätzen der Preise von Objekten zeigten. Bei Patient_innen mit linksfrontaler Lobektomie traten hierbei keine Schwierigkeiten auf. Demgegenüber stehen Studien, welche auch eine Beteiligung der linken Hemisphäre an der Fähigkeit des Schätzens darstellen (Maquet et al., 1996; Rubia, Schuri, Cramon \& Poeppel, 1997; Upton \& Thompson, 1996; Woods et al., 2006).

Tsujii, Sakatani, Masuda, Akiyama und Watanabe (2011) liefern mithilfe der „dual-process theory of reasoning" einen Erklärungsansatz dafür, inwieweit die Schätzfunktion durchaus stärker in der rechten Hemisphäre lokalisiert sein könnte. Das menschliche Denken basiert demnach auf zwei unterschiedlichen Prozessen: einem heuristischen und einem analytischen Prozess. Heuristische Prozesse sind schnell, automatisch und von bestimmten bestehenden Überzeugungen oder Wissen abhängig, wohingegen analytisches Schlussfolgern als langsam, sequenziell und deduktiv charakterisiert wird. Neuroanatomisch betrachtet wird der linke inferiore Frontallappen mit dem heuristischen System in Zusammenhang gebracht, während der bilaterale superiore Parietallappen besonders bei analytischen Denkprozessen relevant ist. Interessant ist, dass der rechte inferiore Frontallappen das linksseitige heuristische System blockieren kann, wenn bei Aufgaben speziell analytische Denkkomponenten benötigt werden (Evans, 2008). Dies legt die Vermutung nahe, dass der rechte Frontallappen auch bei der Funktion des kognitiven Schätzens eine besondere Rolle spielen könnte (Osherson et al., 1998).

Die vorliegende Studie diente der Prüfung, in welchem Ausmaß Störungen des Schätzens bei Patient_innen mit fokaler Epilepsie vorliegen. Besonderes Augenmerk wurde auf Patient_innen mit rechtshemisphärischen Epilepsien im Frontal- und Temporallappen gelegt, da hier gemäß der Datenlage besonders ausgeprägte Schätzdefizite im Vergleich zu Patient_innen mit linkshemisphärischer Epilepsie zu erwarten sind. Ergänzend sollte herausgefunden werden, welchen Anteil pathologische Veränderungen des Hippocampus in beiden Hemisphären am Auftreten von Schätzdefiziten haben.

\section{Methode}

\section{Stichprobe}

In die Studie wurden Daten von insgesamt 176 Personen einbezogen. Die Experimentalgruppe bestand aus 109 Epilepsiepatient_innen (52 Frauen und 57 Männer) des Erlanger Epilepsiezentrums (EZE) mit diagnostizierten Frontal- oder Temporallappenepilepsien (basierend auf EEG- und MRT-Untersuchungen sowie einer ausführlichen neuropsychologischen Diagnostik) und ohne vorherige Operationen am Gehirn. Das Durchschnittsalter der Patientengruppe betrug 37.4 Jahre bei einer Altersspanne von 16 bis 69 Jahren. Bei allen Patient_innen konnte die Epilepsie ursächlich durch einen spezifischen Läsionsbefund (z. B. Tumor, Hippocampussklerose) erklärt werden. Die Untersuchungen erfolgten im Rahmen eines ca. 1-wöchigen stationären Aufenthalts zur präoperativen Epilepsiediagnostik, um neben der Lokalisation des epileptischen Fokus eine Abschätzung bezüglich der Operabilität der Epilepsie zu erlangen.

Eingehende neuropsychologische Untersuchungen sind ein wesentlicher Bestandteil der präoperativen Epilepsiediagnostik am EZE. Neben umfangreichen Untersuchungen von Gedächtnisfunktionen (u.a. verbale und figurale Gedächtnisverarbeitung), Sprachfunktionen (u.a. Benennen, Wortflüssigkeit), Wahrnehmungsfunktionen (u.a. räumlich konstruktive Gestaltverarbeitung, Objekterkennung) sowie Aufmerksamkeits-Exekutivfunktionen werden ebenfalls emotional-affektive Störungen erfasst. In der präoperativen Diagnostik dienen die Ergebnisse der Abschätzung des individuellen Risikos kognitiver Beeinträchtigungen im Falle eines operativen Eingriffs am Gehirn und zum anderen der Bildung einer Lokalisationshypothese hinsichtlich der Verortung des epileptischen Fokus. Daneben leisten die neuro- 
psychologischen Untersuchungen prä- und postoperativ einen unverzichtbaren Beitrag zur Evaluation der operativen Methoden.

Um ein möglichst differenziertes Bild bezüglich des Vorhandenseins von Schätzdefiziten bei fokalen Epilepsien zu erlangen, wurden in der vorliegenden Studie sechs Lokalisationsgruppen gebildet. Die Einteilung betraf zunächst die hemisphärielle Zuordnung (linke, rechte Hemisphäre). Weiterhin wurden entsprechend Patient_innen mit linksfrontaler und rechtsfrontaler Lokalisation der Epilepsie gruppiert. Da bei Temporallappenepilepsien aufgrund der funktionellen Spezialisierungen (v.a. Gedächtnisverarbeitung) in besonderem Maße relevant ist, ob es sich um eine primär neokortikale oder mesiale (Einbeziehung des Hippocampus) Epilepsie handelt, wurde diese Fokusgruppe zweifach gruppiert (neokortikale vs. mesiale Lokalisation). Entsprechend ergaben sich insgesamt sechs Fokusgruppen: linkstemporal-mesial (LT-mes), linkstemporal-neokortikal (LT-neo), rechtstemporal-mesial (RTmes), rechtstemporal-neokortikal (RT-neo), linksfrontal (LF) und rechtsfrontal (RF).

Die Kontrollgruppe bestand aus 67 Personen (38 Frauen und 29 Männer) ohne Epilepsieerkrankung. Ihr Altersdurchschnitt lag bei 32.1 Jahren $(S D=14.89)$. Aus Tabelle 1 können die sozidemografischen und klinischen Daten der Patienten- und Kontrollgruppe entnommen werden. Die Testungen erfolgten im Zeitraum Dezember 2016 bis Dezember 2019.

\section{Studiendesign}

Die Studie wurde von der lokalen medizinischen Ethikkommission der Friedrich-Alexander-Universität Erlangen-Nürnberg genehmigt (Nummer 344_15 B) und im Einverständnis mit den Ethiknormen der World Medical Association (Deklaration von Helsinki) für Experimente mit Menschen durchgeführt.

In dieser Studie wurde ein experimentelles Querschnittdesign gewählt. Die unabhängige Variable stellte die Lokalisation der Epilepsie (LF, RF, LT-mes, LT-neo, RT-mes, RT-neo) dar und die abhängige Variable die Fähigkeit des kognitiven Schätzens.

\section{Erhebungsinstrumente}

Zur Untersuchung des Zusammenhangs zwischen der Fähigkeit des kognitiven Schätzens und der hemisphärischen Lokalisation dieser im Gehirn wurde der Test zum kognitiven Schätzen (TKS; Brand et al., 2002) verwendet. Dieser umfasst die vier Dimensionen Größe, Gewicht, Anzahl und Zeit, zu denen jeweils vier Schätzaufgaben gehören. Ein Beispielitem für die Dimension Zeit lautet: „Wie lange dauert eine morgendliche Dusche?" Insgesamt sind pro Dimension vier Punkte zu erreichen, woraus sich nach dem additiven Prinzip ein Maximalscore von 16 Punkten ergibt. Die interne Konsistenz (Cronbachs Al-

Tabelle 1. Soziodemografische und klinische Daten der Stichproben

\begin{tabular}{|c|c|c|c|c|c|c|c|}
\hline & $\begin{array}{l}\text { LF } \\
(n=15)\end{array}$ & $\begin{array}{l}\mathrm{RF} \\
(n=20)\end{array}$ & $\begin{array}{l}\text { LT-mes } \\
(n=28)\end{array}$ & $\begin{array}{l}\text { LT-neo } \\
(n=16)\end{array}$ & $\begin{array}{l}\text { RT-mes } \\
(n=17)\end{array}$ & $\begin{array}{l}\text { RT-neo } \\
(n=13)\end{array}$ & $\begin{array}{l}\mathrm{KG} \\
(n=67)\end{array}$ \\
\hline Geschlecht weiblich (\%) & $6(40)$ & $11(55)$ & $8(28.6)$ & $8(50)$ & $11(64.7)$ & $8(61.5)$ & $38(56.7)$ \\
\hline Alter* (SD) & $31.1(10.6)$ & $32.4(12.4)$ & $43.1(11.7)$ & $35.5(15.4)$ & $41.9(14.9)$ & $36.8(14.2)$ & $32.1(14.9)$ \\
\hline Rechtshänder (\%) & $14(93.3)$ & $18(90.0)$ & $24(85.7)$ & $14(87.5)$ & $14(82.4)$ & $11(84.6)$ & $67(100)$ \\
\hline $\mathrm{VIQ}(S D)$ & $94.6(10.04)$ & $99.3(13.75)$ & 98. (9.59) & $101.9(11.09)$ & $97.1(14.51)$ & $103.4(14.77)$ & \\
\hline Beginn** (SD) & $17.9(10.83)$ & $15.8(11.96)$ & $25.0(16.11)$ & $28.5(15.00)$ & $18.7(8.76)$ & $28.1(18.66)$ & \\
\hline Dauer* (SD) & $13.1(13.15)$ & $16.5(11.85)$ & $17.6(13.74)$ & $7.1(8.92)$ & $23.2(14.25)$ & $8.6(7.92)$ & \\
\hline Anzahl*** (SD) & $4.9(9.49)$ & $8.7(11.75)$ & $5.4(8.26)$ & $5.1(10.18)$ & $8.9(13.20)$ & $3.9(8.33)$ & \\
\hline \multicolumn{8}{|l|}{ Pathologie } \\
\hline HS (\%) & 0 & 0 & $18(64.3)$ & 0 & $12(70.6)$ & 0 & \\
\hline Tumor (\%) & $4(26.7)$ & $3(15)$ & $7(25)$ & $6(37.5)$ & $3(17.6)$ & $4(30.8)$ & \\
\hline CD (\%) & $8(53.3)$ & $10(50)$ & 0 & $2(12.5)$ & 0 & $1(7.7)$ & \\
\hline Trauma (\%) & $1(6.7)$ & 0 & 0 & 0 & 0 & $1(7.7)$ & \\
\hline Gefäßfehlbildung (\%) & 0 & $1(5)$ & 0 & $7(43.8)$ & 0 & $4(30.8)$ & \\
\hline Sonstige (\%) & $2(13.3)$ & $6(30)$ & $3(10.7)$ & $1(6.3)$ & $2(11.8)$ & $3(23.1)$ & \\
\hline
\end{tabular}

Anmerkungen: LF = linksfrontal; RF = rechtsfrontal; LT-mes = linkstemporal-mesial; LT-neo = linkstemporal neokortikal; RT-mes = rechtstemporal-mesial; RT-neo = rechtstemporal-neokortikal; $K G=$ Kontrollgruppe; $n=$ Anzahl der Personen; $S D=$ Standardabweichung; VIQ = verbaler Intelligenzquotient; HS = Hippocampussklerose; CD = Kortikale Dysplasie; * Gruppendurchschnitt in Jahren; **Durchschnittsalter in Jahren; ***Anfälle/Monat. 
pha) des TKS beträgt $\alpha=0.77$ (Pedraza, Sachs, Ferman, Rush \& Lucas, 2011).

Deskriptive Daten, welche das Alter, das Geschlecht, die Händigkeit, die Ausprägung des sprachbezogenen Intelligenzniveaus (Mehrfachwahl-Wortschatz-Intelligenztest [MWT-B]; Lehrl, 1999), das Alter bei Erkrankungsbeginn, die Erkrankungsdauer, die Anzahl der Anfälle pro Monat und die Art der Pathologie umfassten, wurden zusätzlich erhoben. Die Händigkeit wurde neben anamnestischen Angaben mit einer Kurzform des Edinburgh Handedness Inventory (Oldfield, 1971) erfasst. Hierzu wurden 12 Fragen gestellt (z. B. Schreiben, Malen, einen Ball werfen), zu denen die jeweilige Handpräferenz angegeben werden sollte (immer links, überwiegend links, keine Präferenz, überwiegend rechts, immer rechts).

\section{Statistische Analysen}

Um zu prüfen, ob statistisch signifikante Gruppenunterschiede im Hinblick auf die Fähigkeit des kognitiven Schätzens vorliegen, wurde eine einfaktorielle ANOVA mit der unabhängigen Variable der Gruppenzugehörigkeit und der abhängigen Variable der Schätzleistung gerechnet. Zudem wurde ein Post-hoc-Test für ungleiche Stichprobengrößen (nach Scheffé) angewendet, um spezifische Gruppenunterschiede zu identifizieren. Potenzielle Einflüsse des Alters und des verbalen Intelligenzniveaus wurden berücksichtigt. Um ein klareres Bild hinsichtlich eines möglichen Einflusses des Intelligenzniveaus und des Alters auf die Schätzleistung zu erhalten, wurde der Zusammenhang ergänzend mittels Korrelationsanalyse geprüft. Für alle statistischen Tests galt das Signifikanzniveau von $\alpha=0.05$. Die Prüfung, inwieweit die einzelnen Testparameter normalverteilt sind oder davon abweichen, erfolgte mittels Kolmogorov-Smirnov-Test.

\section{Ergebnisse}

Mittels Korrelationsanalyse konnte kein Einfluss des verbalen Intelligenzquotienten auf die Schätzfähigkeit der Patientengruppen festgestellt werden (Pearson Korrelationskoeffizient: $p=.22$ ). Ebenfalls ergab sich bezogen auf das Alter kein signifikantes Ergebnis $(p=.84)$, wodurch ein negativer Effekt aufgrund altersbedingter Veränderungen ausgeschlossen werden konnte.

In Bezug auf die Hypothese, dass Beeinträchtigungen der Schätzfunktion insbesondere bei rechtshemisphärischen Epilepsien auftreten, wurden in der statistischen Analyse folgende Ergebnisse erzielt: Die einfaktorielle ANOVA verwies auf einen signifikanten
Unterschied innerhalb der Gruppen $(F[6,58,45]=9.93)$. Der Test auf Homogenität der Varianzen ergab ein nicht signifikantes Ergebnis (Levene-Test: $p=0.32$ ). In der Prüfung auf Normalverteilung der Ergebnisse mittels Kolmogorov-Smirnov-Test zeigte sich einzig für die RTmes- $(p=.01)$ und RT-neo- $(p=.03)$ Gruppen ein signifikantes Ergebnis.

Auf dem Signifikanzniveau von $\alpha=0.05$ ergaben sich in den Post-hoc-Tests (nach Scheffé) bezüglich der Patientengruppen signifikante Unterschiede in der Schätzfähigkeit zwischen den Gruppen RF und LT-mes ( $p=.05)$, zwischen RF und RT-neo $(p=.01)$, zwischen LT-mes und RT-mes $(p=.003)$, zwischen LT-neo und RT-mes $(p=.001)$, und zwischen RT-mes und RT-neo $(p=.001)$. Für die Gruppe LF wurde ein knapp nicht signifikantes Ergebnis im Vergleich zu RT-mes festgestellt $(p=.07)$.

Die Kontrollgruppe wies signifikante Unterschiede $\mathrm{zu}$ den Gruppen RF ( $p=.002)$ und RT-mes ( $p=.000)$ auf. Die linkshemisphärischen Läsionsgruppen sowie die Gruppe RT-neo hoben sich nicht signifikant von der Kontrollgruppe ab. Anhand der Ergebnisse wird eine vorherrschende Beteiligung der rechten Hemisphäre an der Fähigkeit des kognitiven Schätzens deutlich.

Beim Vergleich der im TKS erzielten $z$-Werte zeigte sich eine defizitäre Schätzleistung bei den Patient_innen mit rechtsfrontalen und rechtstemporal-mesialen Epilepsieherden (siehe Tabelle 2). Zur Verdeutlichung der Unterschiede bildet Abbildung 1 die gruppenspezifische Leistung im Schätztest ab.

Um die gewonnenen Ergebnisse - hier insbesondere die vergleichsweise stärkere rechtshemisphärische Lateralisation der Schätzfunktion - auf Einzelfallebene zu illustrieren, werden im Folgenden die kernspintomografischen Bilder von zwei Patientinnen präsentiert. Sie sollen zeigen, dass es einen klaren Zusammenhang zwischen Neuropathologie, hemisphärieller Lokalisation und funktionellem Schätzdefizit gibt. Bei einer Patientin ergab sich in der Bildgebung der Befund einer im rechten Frontallappen lokalisierten fokalen kortikalen Dysplasie (Abbildung 2). Bei einer anderen Patientin zeigte sich eine typische Verschmächtigung des rechten Hippocampus im Sinne einer Hippocampussklerose rechts (Abbildung 3). Beide Patientinnen wiesen analog zu den Gruppenvergleichen besonders ausgeprägte Schätzdefizite im TKS auf (jeweils $z=3.3$ ).

\section{Diskussion}

Den Erwartungen entsprechend zeigten sich Defizite in der Fähigkeit des kognitiven Schätzens speziell bei Patient_innen mit rechtshemisphärischen Läsionen. Entspre- 
Tabelle 2. Übersicht der im TKS erzielten z-Werte nach Untergruppe

\begin{tabular}{llll}
\hline Gruppe & $n$ & $M$ & $S D$ \\
\hline LF & 15 & -0.35 & 0.97 \\
RF & 20 & -1.19 & 1.02 \\
LT-mes & 28 & -0.16 & 1.15 \\
LT-neo & 16 & 0.29 & 0.79 \\
RT-mes & 17 & -1.55 & 0.98 \\
RT-neo & 13 & 0.23 & 0.70 \\
KG & 67 & 0.00 & 1.00 \\
\hline
\end{tabular}

Anmerkungen: $n=$ Anzahl der Personen; $M=$ Mittelwert; $S D=$ Standardabweichung; LF = linksfrontal; RF = rechtsfrontal; LT-mes = linkstemporalmesial; $\mathrm{LT}$-neo = linkstemporal neokortikal; RT-mes = rechtstemporal-mesial; $\mathrm{RT}-$ neo $=$ rechtstemporal-neokortikal; $K G=$ Kontrollgruppe.

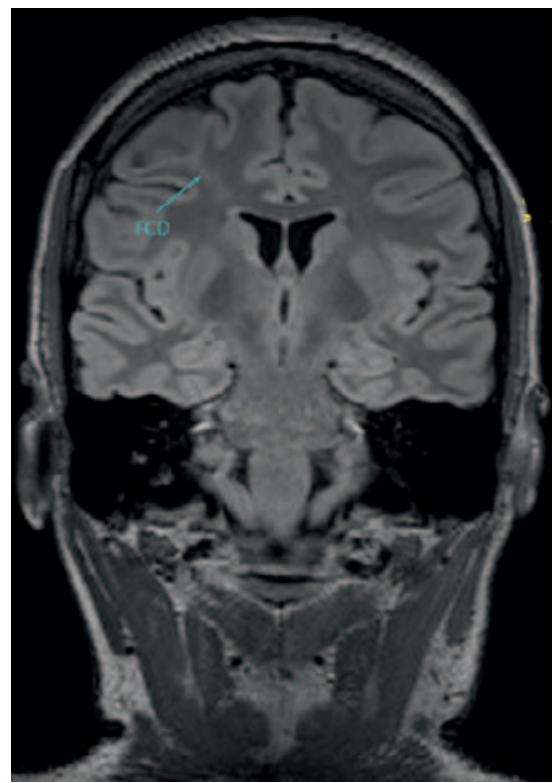

Abbildung 2. MRT (FLAIR cor.) einer 24-jährigen Patientin, Erkrankungsbeginn: 16. Lebensjahr; Ätiologie: Transmanteldysplasie vom Gyrus frontalis medius rechts bis an den Seitenventrikel (FCD).

chend der Gruppierung betraf dies speziell Patient_innen mit fokaler Epilepsie im rechten Frontallappen bzw. bei vorliegender Hippocampussklerose rechts.

Die unterdurchschnittlichen Ergebnisse im TKS bei Patient_innen mit rechtstemporalen Hirnläsionen - Patient_innen mit neokortikalen temporalen Lokalisationen zeigten hier keine Auffälligkeiten - lassen eine starke Be-

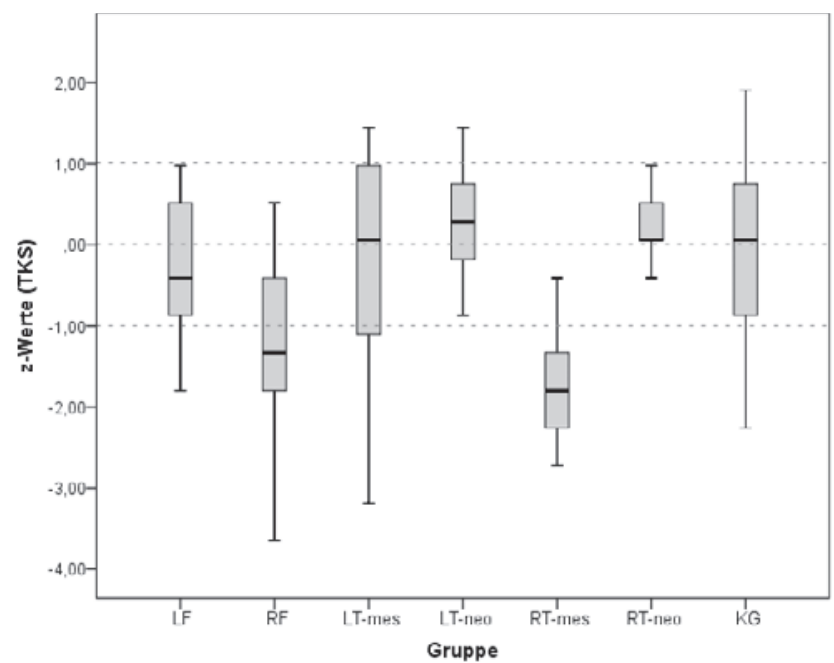

Abbildung 1. Erzielte $z$-Werte im TKS nach Untergruppen sortiert (Abkürzungen: $K G=$ Kontrollgruppe; $L F=$ linksfrontal; LT-mes = linkstemporal-mesial; LT-neo = linkstemporal neokortikal; RF = rechtsfrontal; RTmes $=$ rechtstemporal-mesial; RT-neo = rechtstemporal-neokortikal).

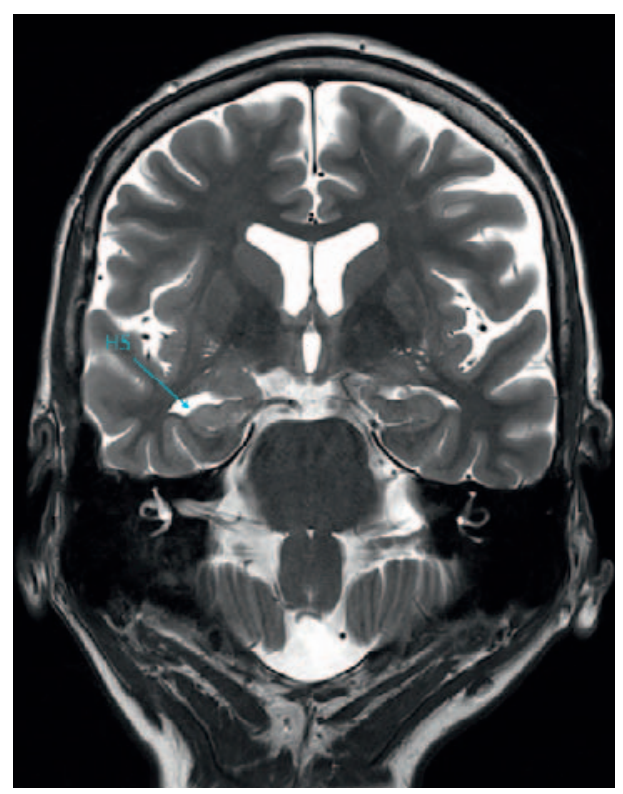

Abbildung 3. MRT (T2 cor.) einer 52-jährigen Patientin, Erkrankungsbeginn: 25. Lebensjahr; Ätiologie: Hippocampussklerose rechts (HS).

teiligung des Hippocampus an der Kontrolle exekutiver Funktionen annehmen. Dieses Ergebnis ist zunächst überraschend, da der Hippocampus in seiner primären funktionellen Zuordnung klassischerweise mit materialspezifischen Gedächtnisprozessen assoziiert wird (Coras et al., 2014). Dennoch lassen sich in der Literatur auch Hinweise auf eine stärkere Involvierung des Hippocam- 
pus bei der Bewältigung von Aufgaben, bei denen ein erhöhtes strategisches Planungsverhalten erforderlich ist, finden (Corkin, 2001). Damit ist der Hippocampus von größerer Bedeutung bei komplexen mentalen Verarbeitungsprozessen als bisher angenommen.

Bislang war zudem nur schwer möglich, insbesondere funktionelle Auffälligkeiten bei rechtsfrontalen Epilepsiefoci mittels neuropsychologischer Testungen präzise zu erfassen. Während eine zielgerichtete Lokalisationsdiagnostik bei linkshemisphärischen Läsionen in Form von verbalen Gedächtnisdefiziten bzw. Sprachstörungen (z. B. Benennen, Wortflüssigkeit) vergleichsweise gut möglich ist (Rosazza et al., 2009; Schwarz \& Pauli, 2009), fehlte bei rechtsfrontalen Schädigungen bislang ein spezifischer Indikator. Für diese Zwecke kann eine Einbeziehung von Schätzaufgaben ein hilfreiches Diagnoseinstrument darstellen.

Auffallend ist, dass in der vorliegenden Studie keine Hinweise auf eine wesentliche linkshemisphärische Beteiligung an der Funktion des Schätzens ersichtlich wurden. Dies steht in Einklang mit bereits erwähnten Studien und demonstriert eine gewisse rechtshemisphärische Dominanz für Schätzprozesse (Maquet et al., 1996; Rubia et al., 1997; Upton \& Thompson, 1996; Woods et al., 2006).

Zusammenfassend ist festzuhalten, dass die Lokalisation des epileptischen Fokus einen deutlichen Effekt auf die Schätzleistung hat. Man erkennt aus den Befunden eine gewisse Rechtslateralisierung der Schätzfähigkeit, die vor allem für den rechten Frontallappen und den rechten mesialen Temporallappen zutrifft. Aufgrund der Größe speziell des Frontallappens sollte zukünftig jedoch noch genauer geprüft werden, welche frontalen Lokalisationen in besonderer Weise zu Schätzdefiziten führen und welche frontalen Bereiche eine vergleichsweise geringere Relevanz für exekutive Prozesse haben.

\section{Bedeutung für die Praxis}

Im Rahmen der neuropsychologischen Epilepsiediagnostik können Beeinträchtigungen in der Fähigkeit des kognitiven Schätzens einen wichtigen Beitrag zur Lokalisation der Epilepsie leisten. In der vorliegenden Studie wiesen insbesondere Patient_innen mit rechtsfrontalen und rechtstemporalen mesialen Läsionen Defizite in diesem Bereich auf. Zur Lokalisation einer fokalen Epilepsie zeigt sich in der neuropsychologischen Diagnostik der Test zum kognitiven Schätzen hilfreich, nicht zuletzt, weil er mit seiner höchstens 10-minütigen Durchführungsdauer eine hohe Testökonomie aufweist.

Daher ist es sinnvoll, im Rahmen der neuropsychologischen Diagnostik neben den Gedächtnis- und Sprachfunktionen ebenfalls die Schätzfähigkeit zu erfassen.

\section{Literatur}

Benke, T., Lehner-Baumgartner, E., Bodner, T., Karner, E. \& Delazer, M. (2007). Neuropsychologie im Rahmen der prächirurgischen Epilepsiediagnostik. Mitteilungen der Österreichischen Sektion der Internationalen Liga gegen Epilepsie, 7, 2-10.

Brand, M., Kalbe, E. \& Kessler, J. (2002). Test zum kognitiven Schätzen:TKS; Manual. Weinheim: Beltz.

Braun, I., Schwarz, M., Walther, K., Stemmler, M., Kasper, B.S. \& Hamer, H. (2018). Functional dissociation between cognitive estimation and object naming in focal temporal and frontal lobe epilepsies. International Journal of Epilepsy, 5, 44-49.

Bunge, S.A. \& Souza, M.J. (2009). Executive function and higherorder cognition: Neuroimaging, 4,111-116.

Coras, R., Pauli, E., Li, J., Schwarz, M., Rossler, K., Buchfelder, M. et al. (2014). Differential influence of hippocampal subfields to memory formation: Insights from patients with temporal lobe epilepsy. Brain, 137, 1945-1957.

Corkin, S. (2001). Beware of frontal lobe deficits in hippocampal clothing. Trends in Cognitive Sciences, 5, 321-323.

D’Aniello, G. E., Scarpina, F., Albani, G., Castelnuovo, G. \& Mauro, A. (2015). Disentangling the relationship between cognitive estimation abilities and executive functions: A study on patients with Parkinson's disease. Neurological Sciences, 36 , $1425-1429$.

Evans, J.S.B. (2008). Dual-processing accounts of reasoning, judgment, and social cognition. Annual Review of Psychology, 59, 255-278.

Helmstaedter, C., Kemper, B. \& Elger, C.E. (1996). Neuropsychological aspects of frontal lobe epilepsy. Neuropsychologia, 34, 399-406.

Hernandez, M.T., Sauerwein, H.C., Jambaqué, I., De Guise, E., Lussier, F., Lortie, A. et al. (2002). Deficits in executive functions and motor coordination in children with frontal lobe epilepsy. Neuropsychologia, 40, 384-400.

Lehrl, S. (1999). Mehrfachwahl-Wortschatz-Intelligenztest: MWTB. Balingen: Spitta.

Lewis, P.A. \& Miall, R.C. (2006). A right hemispheric prefrontal system for cognitive time measurement. Behavioural Processes, 71, 226-234.

Maquet, P., Lejeune, H., Pouthas, V., Bonnet, M., Casini, L., Macar, F. et al. (1996). Brain activation induced by estimation of duration: A PET study. Neuroimage, 3, 119-126.

Morin, C., Guigot, J., Manai, R., Vu, P., Guérin, N., Samson, Y. et al. (2005). Impairment in clock-time estimation following right hemisphere ischemic damage. Cognitive Brain Research, 22 305-307.

Müller, S.V., Klein, T. et al. (2019). Diagnostik und Therapie von exekutiven Dysfunktionen bei neurologischen Erkrankungen, S2eLeitlinie, 2019. In Deutsche Gesellschaft für Neurologie (Hrsg.), Leitlinien für Diagnostik und Therapie in der Neurologie. Verfügbar unter www.dgn.org/leitlinien

Oldfield, R.C. (1971). The assessment and analysis of handedness: The Edinburgh inventory. Neuropsychologia, 9, 97-113.

Osherson, D., Perani, D., Cappa, S., Schnur, T., Grassi, F. \& Fazio, F. (1998). Distinct brain loci in deductive versus probabilistic reasoning. Neuropsychologia, 36, 369-376.

Pedraza, O., Sachs, B.C., Ferman, T.J., Rush, B.K. \& Lucas, J.A. (2011). Difficulty and discrimination parameters of Boston Naming Test items in a consecutive clinical series. Archives of Clinical Neuropsychology, 26, 434-444.

Rosazza, C., Minati, L., Ghielmetti, F., Maccagnano, E., Erbetta, A., Villani, F. et al. (2009). Engagement of the medial temporal lobe in verbal and nonverbal memory: Assessment with functional MR imaging in healthy subjects. American Journal of Neuroradiology, 30, 1134-1141. 
Rubia, K., Schuri, U.V., Cramon, D.Y. \& Poeppel, E. (1997). Time estimation as a neuronal network property: A lesion study. Neuroreport, 8, 1273-1276.

Schwarz, M. \& Pauli, E. (2009). Postoperative speech processing in temporal lobe epilepsy: Functional relationship between object naming, semantics and phonology. Epilepsy \& Behavior, 16, 629-633.

Shallice, T. (1982). Specific impairments of planning. Philosophical Transactions of the Royal Society B, 298, 199-209.

Shallice, T. \& Evans, M.E. (1978). The involvement of the frontal lobes in cognitive estimation. Cortex, 14, 294-303.

Smith, M.L. \& Milner, B. (1984). Differential effects of frontal-lobe lesions on cognitive estimation and spatial memory. Neuropsychologia, 22, 697-705.

Tsujii, T., Sakatani, K., Masuda, S., Akiyama, T. \& Watanabe, S. (2011). Evaluating the roles of the inferior frontal gyrus and superior parietal lobule in deductive reasoning: An rTMS study. Neuroimage, 58, 640-646.

Upton, D. \& Thompson, P.J. (1996). General neuropsychological characteristics of frontal lobe epilepsy. Epilepsy Research, 23, $169-177$.

van Rijckevorsel, K. (2006). Cognitive problems related to epilepsy syndromes, especially malignant epilepsies. Seizure, 15, 227-234.

Woods, A.J., Mennemeier, M., Garcia-Rill, E., Meythaler, J., Mark, V.W., Jewel, G. R. et al. (2006). Bias in magnitude estimation following left hemisphere injury. Neuropsychologia, 44, 1406-1412.

\section{Historie}

Eingereicht: 13. Januar 2021

Akzeptiert: 19. März 2021

\section{Ethische Richtlinien}

Die Studie wurde mit der Zustimmung der zuständigen Ethikkommission, im Einklang mit dem nationalen Recht sowie gemäß der Deklaration von Helsinki durchgeführt. Die Teilnahme an den Testungen erfolgte freiwillig. Ein Rückschluss auf persönliche Merkmale der Proband_innen ist nicht möglich.

\section{Förderung}

Open-Access-Veröffentlichung ermöglicht durch

Friedrich-Alexander-Universität Erlangen-Nürnberg.

\section{Interessenkonflikt}

Bei der Durchführung und Ausarbeitung der vorliegenden Studie lag keinerlei Interessenkonflikt vor.

\section{Dr. Michael Schwarz}

Neurologische Universitätsklinik Erlangen

Epilepsiezentrum

Schwabachanlage 6

91052 Erlangen

Deutschland

Michael.Schwarz@uk-erlangen.de 